\title{
Preparing photocatalytic paper with improved catalytic activity by in situ loading poly-dopamine on cellulose fibre
}

\author{
ZHUOZHUO QIN, WENXIA LIU*, HUABIN CHEN, JUN CHEN, HUILI WANG \\ and ZHAOPING SONG \\ Key Laboratory of Pulp and Paper Science and Technology (Ministry of Education), Qilu University of Technology \\ (Shandong Academy of Science), Jinan, Shandong 250353, People's Republic of China \\ *Author for correspondence (liuwenxia@qlu.edu.cn)
}

MS received 4 January 2018; accepted 22 July 2018; published online 4 March 2019

\begin{abstract}
Photocatalytic paper is a composite photocatalyst generally prepared by loading semiconductors, such as titanium dioxide $\left(\mathrm{TiO}_{2}\right)$ nanoparticles on paper, and attracts sustaining interests. In this paper, poly-dopamine (PDA) was loaded on cellulose fibre by in situ oxidative polymerization of dopamine. The PDA-loaded cellulose fibre (PLCF) was used to make $\mathrm{TiO}_{2}$ photocatalytic paper together with pristine cellulose fibre $(\mathrm{CF})$ and $\mathrm{TiO}_{2}$ nanoparticles to improve the performance of the photocatalytic paper. The loading of PDA on CF and the introduction of $\mathrm{TiO}_{2}$ nanoparticles in paper were verified by infrared spectroscopy and X-ray diffraction technique, respectively. As observed by scanning electron microscopy, the introduction of PLCF significantly improved the distribution of $\mathrm{TiO}_{2}$ nanoparticles in paper by forming a membranous substance. The photocatalytic performance of the as-prepared photocatalytic paper was analysed by decomposing methyl orange under UV light irradiation. It was confirmed that the occurrence of PLCF significantly improved the photocatalytic performance of the photocatalytic paper. The paper with the highest photocatalytic activity was prepared using a PLCF-to-CF mass ratio of 1:1. The photocatalytic paper with PLCF is stable in the process of repeated application. This work provided a new approach to prepare photocatalytic paper with improved photocatalytic activity.
\end{abstract}

Keywords. Photocatalytic paper; poly-dopamine; $\mathrm{TiO}_{2}$ nanoparticles; photocatalysis; antibacterial activity.

\section{Introduction}

Owing to the requirement of environmental protection and utilization of renewable energy sources, the harvesting of sunlight for environmental remediation has attracted extensive attention, and a number of potential photocatalysts, such as titanium dioxide $\left(\mathrm{TiO}_{2}\right)$ nanoparticles have been reported widely [1-3]. However, during the repeated photocatalytic process, there is a loss of photocatalysts if they are used in the form of nanoparticles. Also, they are difficult to recycle [1]. Using cellulose material as a carrier, photocatalysts can be introduced into the unique network structure of cellulose paper, and a novel cellulose-based functional material is prepared [4]. This kind of photocatalytic paper not only reduces the loss of nanophotocatalyst, but may also increase the photocatalytic efficiency, showing a better photocatalytic performance $[5,6]$. Meanwhile, paper-shaped photocatalytic materials may have a wider applications as decorating or construction materials in rooms.

$\mathrm{TiO}_{2}$ has attracted considerable attention due to its strong photocatalytic activity, robust chemical stability under acidic and oxidative environments, high electrical and optical properties, non-toxicity and low production cost [7]. It has been used in numerous fields, such as photocatalysis [8], optoelectronics, dye-sensitized solar cells [9], biomedical devices
[10], gas sensors [11] and water purification [12]. Among the three most abundant crystallographic forms of $\mathrm{TiO}_{2}$ (anatase, rutile and brookite), the anatase form is mainly used in photocatalysis and solar energy conversion due to its higher charge-carrier mobility and greater density of surface hydroxyls $[13,14] . \mathrm{TiO}_{2}$ nanoparticles composed of about $80 \%$ of anatase and $20 \%$ of rutile, are the most studied photocatalytic materials and exhibit excellent photocatalytic performance under UV light irradiation owing to the broad band gap that matches the high photonic energy of UV light. $\mathrm{TiO}_{2}$ photocatalytic paper as the most widely studied photocatalytic paper is generally formed by loading $\mathrm{TiO}_{2}$ nanoparticles on cellulose fibres (CF) before paper manufacture [4]. To improve the photocatalytic performance or durability of photocatalytic paper, inorganic carriers such as zeolite [15] or carbon fibre [16] are generally used as supporting matrix for $\mathrm{TiO}_{2}$ nanoparticles.

Poly(dopamine) (PDA) is a biocompatible polymer. PDA and PDA-derived materials have drawn plenty of attention since 2007 when they were first reported as a coating material [17]. PDA could be formed on a wide range of inorganic and organic materials by in situ spontaneous oxidative polymerization of dopamine in alkaline solution at room temperature. The in situ spontaneous polymerization of dopamine provides the advantages of one-step surface functionalization with 


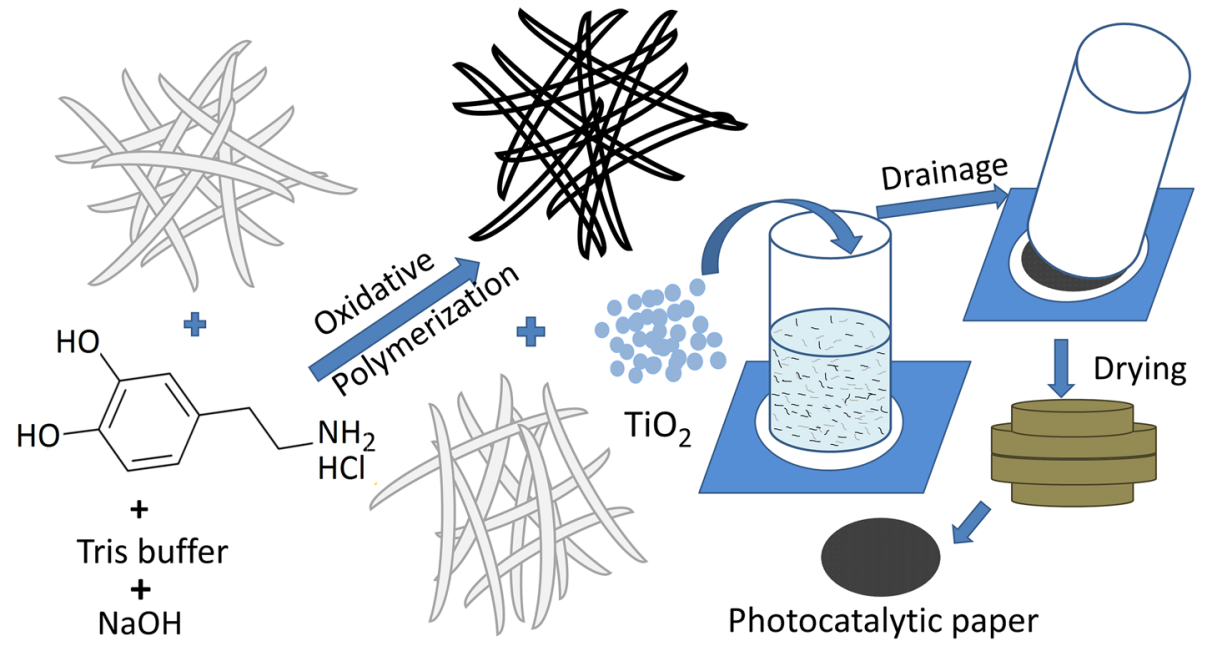

Figure 1. Schematic diagram for manufacturing of photocatalytic paper loaded with PDA.

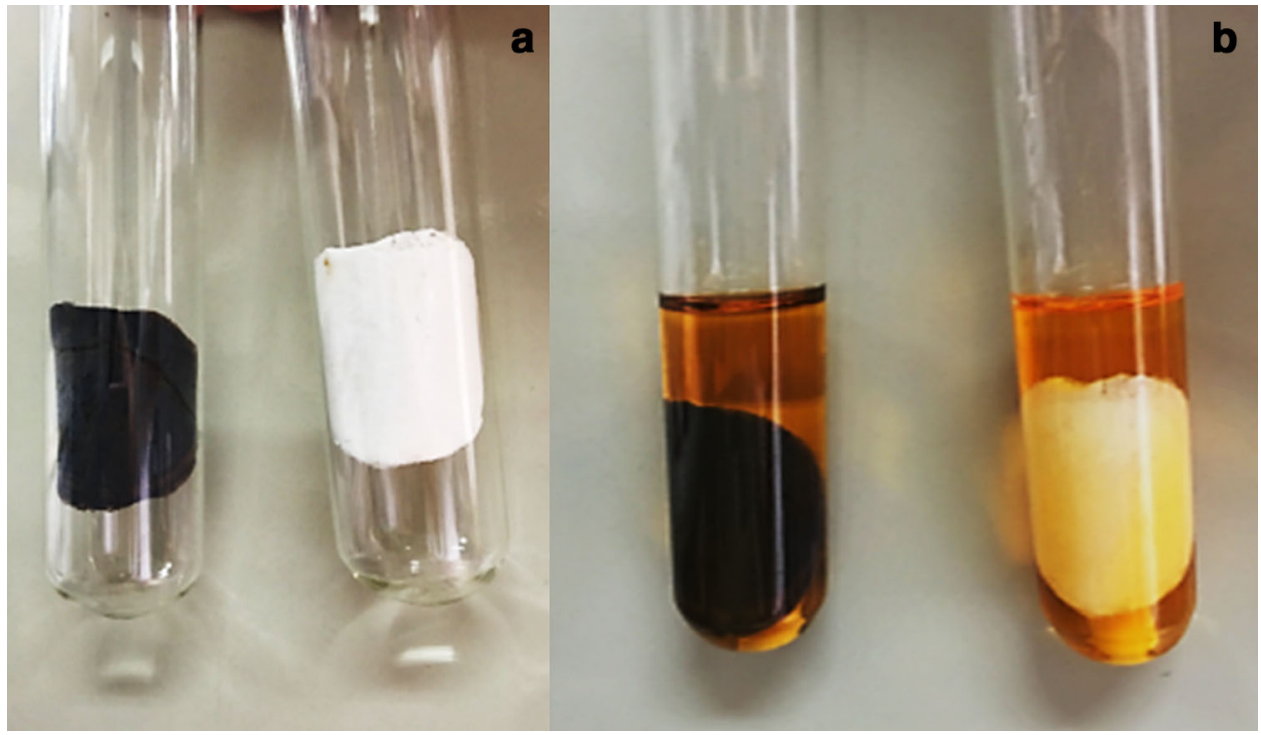

Figure 2. Photographs of test tubes containing photocatalytic paper with a diameter of $30 \mathrm{~mm}$ (a) before and (b) after the addition of $20 \mathrm{ml}$ of MO aqueous solution with a concentration of $20 \mathrm{mg}^{-1}$. The white paper sample is the photocatalytic paper without loading PDA. The black paper is the photocatalytic paper loaded with PDA, where the mass ratio of PLCF-CF is 1:1.

simple ingredients, mild reaction conditions and applicability to various types of materials. PDA displays many excellent electrical, magnetic and optical properties and most importantly, it possesses high specific surface area, superior biocompatibility and biodegradability [18].

In this paper, PDA was loaded on CF by in situ oxidative polymerization of dopamine. The as-prepared PDA-loaded cellulose fibre (PLCF) was made into a photocatalytic paper together with pristine $\mathrm{CF}$ and $\mathrm{TiO}_{2}$ nanoparticles using the papermaking technique. The photocatalytic activity of the photocatalytic paper was tested by means of the degradation of methyl orange (MO). It was found that the introduction of PLCF enhanced the photocatalytic effect of $\mathrm{TiO}_{2}$ photocatalytic paper under UV irradiation.

\section{Experimental}

\subsection{Materials}

$\mathrm{TiO}_{2}$ nanoparticles (ca. $80 \%$ anatase and $20 \%$ rutile) as a commercial product Titania P25 was purchased from Evonik 
Degussa $\mathrm{GmbH}$. Sodium hydroxide $(\mathrm{NaOH})$, hydrochloric acid, trihydroxymethylaminomethane (Tris) and dopamine hydrochloride were all of analytical grade and purchased from Sinopharm Chemical Reagents Co. Ltd. Bleached softwood pulp with beating degree of about $43^{\circ} \mathrm{SR}$ and polyaminoamide-epichlorohydrin (PAE, a paper wet strength agent) were kindly provided by a paper mill from Shandong Province, China. The MO used for this study was purchased from Tianjin Bodi Reagent Factory. All solutions were prepared using deionized water.

\subsection{Preparation and characterization of photocatalytic paper}

Figure 1 shows the schematic diagram for the manufacturing process of photocatalytic paper loaded with PDA. PLCF was first prepared by in situ oxidative polymerization of dopamine with softwood pulp before paper making. In a typical process, $0.121 \mathrm{~g}$ trishydroxymethylaminomethane was dissolved in $100 \mathrm{ml}$ deionized water to obtain $0.01 \mathrm{~mol}^{-1}$ Tris buffer solution, then $0.2 \mathrm{~g}$ dopamine hydrochloride was dissolved in the Tris buffer solution. After $\mathrm{pH}$ of the solution was adjusted with $\mathrm{NaOH}$ solution to $8.5,1.0 \mathrm{~g}$ softwood pulp (i.e., pristine $\mathrm{CF}$ ) was added. The mixture was warmed to $30^{\circ} \mathrm{C}$ under magnetic stirring, reacted for $24 \mathrm{~h}$, and washed thoroughly with deionized water by multiple rounds of centrifugation to obtain the PLCF.

The as-prepared PLCF, pristine $\mathrm{CF}$ and $\mathrm{TiO}_{2}$ nanoparticles were proportionally dispersed into deionized water to form a $1 \%$ pulp suspension. The mass ratio of $\mathrm{TiO}_{2}$ nanoparticles to total CF is fixed at 1:5. The suspension was treated in a defibrizer for $1 \mathrm{~min}$, then, $1.0 \mathrm{wt} \%$ of PAE based on ovendry fibre was added within another $1 \mathrm{~min}$. Handsheets with a base weight of $\sim 90 \mathrm{~g} \mathrm{~m}^{-2}$ were made by filtering the suspension on a sub-microporous filter paper through a vacuum filter. After being pressed at $350 \mathrm{kPa}$ for $5 \mathrm{~min}$, the wet sheets were dried in an oven at $105^{\circ} \mathrm{C}$ for $10 \mathrm{~min}$. $\mathrm{TiO}_{2}$ photocatalytic paper without PLCF was also prepared as blank paper. The presence of $\mathrm{TiO}_{2}$ nanoparticles in the as-prepared paper was analysed using $\mathrm{X}$-ray diffraction (XRD) technique. The XRD pattern was recorded on a Bruker D8 Advance powder $\mathrm{X}$-ray diffractometer with $\mathrm{CuK} \alpha$ radiation $(\lambda=0.15406 \mathrm{~nm})$. The loading of PDA on CF was observed either by colour variation or by optical microscopy (BK200, Chongqing Optical Instrument Co. Ltd.).

\subsection{Photocatalytic activity test}

The photocatalytic performance of photocatalytic papers was evaluated by testing the degradation rate of MO under UV light irradiation in the presence of paper. In a typical experiment, circular photocatalytic paper with a diameter of $30 \mathrm{~mm}$ was adhered to the inner wall of a $50 \mathrm{ml}$ quartz test tube before $20 \mathrm{ml}$ of $\mathrm{MO}$ aqueous solution with a concentration of $20 \mathrm{mg} \mathrm{l}^{-1}$ was added as shown in figure 2. Both adsorp-

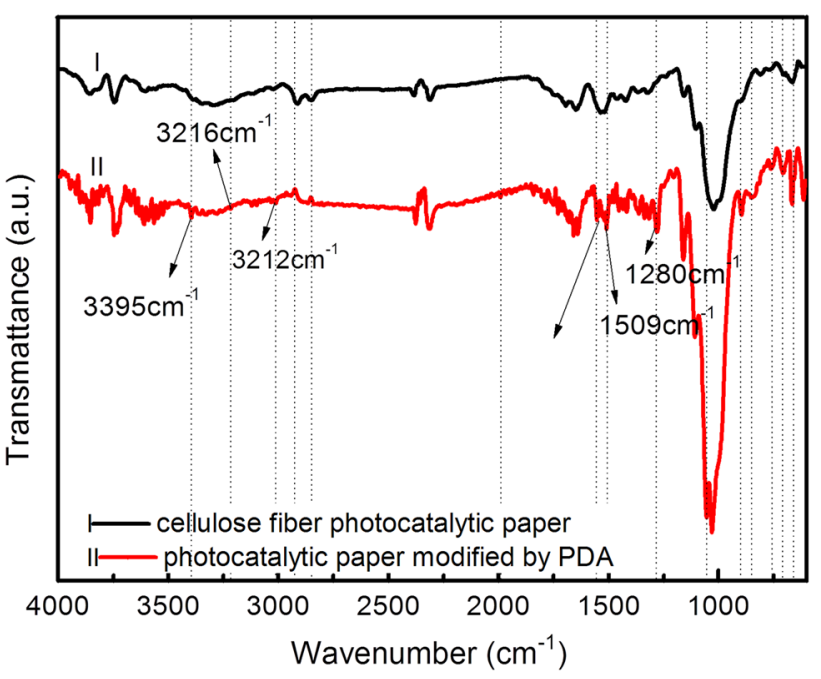

Figure 3. FTIR spectra of photocatalytic papers (I) without PDA and (II) with PLCF, where the mass ratio of PLCF-CF is 1:1.

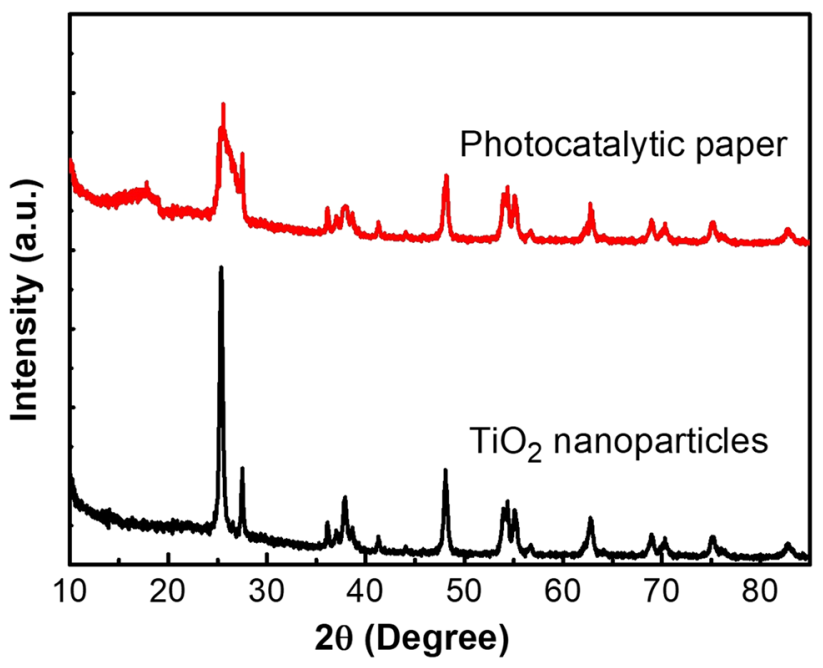

Figure 4. XRD patterns of $\mathrm{TiO}_{2}$ nanoparticles and photocatalytic paper with a PLCF-CF mass ratio of $1: 1$.

tion and photocatalytic activity tests were carried out for comparison $[19,20]$. In the adsorption test, the test tube was placed in the dark for observing the adsorption of MO on the photocatalytic paper. In the photocatalytic activity test, the test tube containing same photocatalytic paper and MO solution was exposed under UV light. A $500 \mathrm{~W}$ mercury lamp with a primary wavelength of $365 \mathrm{~nm}$ was chosen as the UV source. The reaction temperature was kept at $25^{\circ} \mathrm{C}$ by circulating cool water. The concentrations of residual MO solution during the reaction period were determined by measuring the absorbance at $465 \mathrm{~nm}$ using a UV-Vis spectrophotometer (Hitachi UV300) [21]. Each aliquot was withdrawn every $30 \mathrm{~min}$. In all the repeated photocatalytic tests, the photocatalytic paper was dried at $60^{\circ} \mathrm{C}$ after washing with deionized water, and used in the next repeated test. 

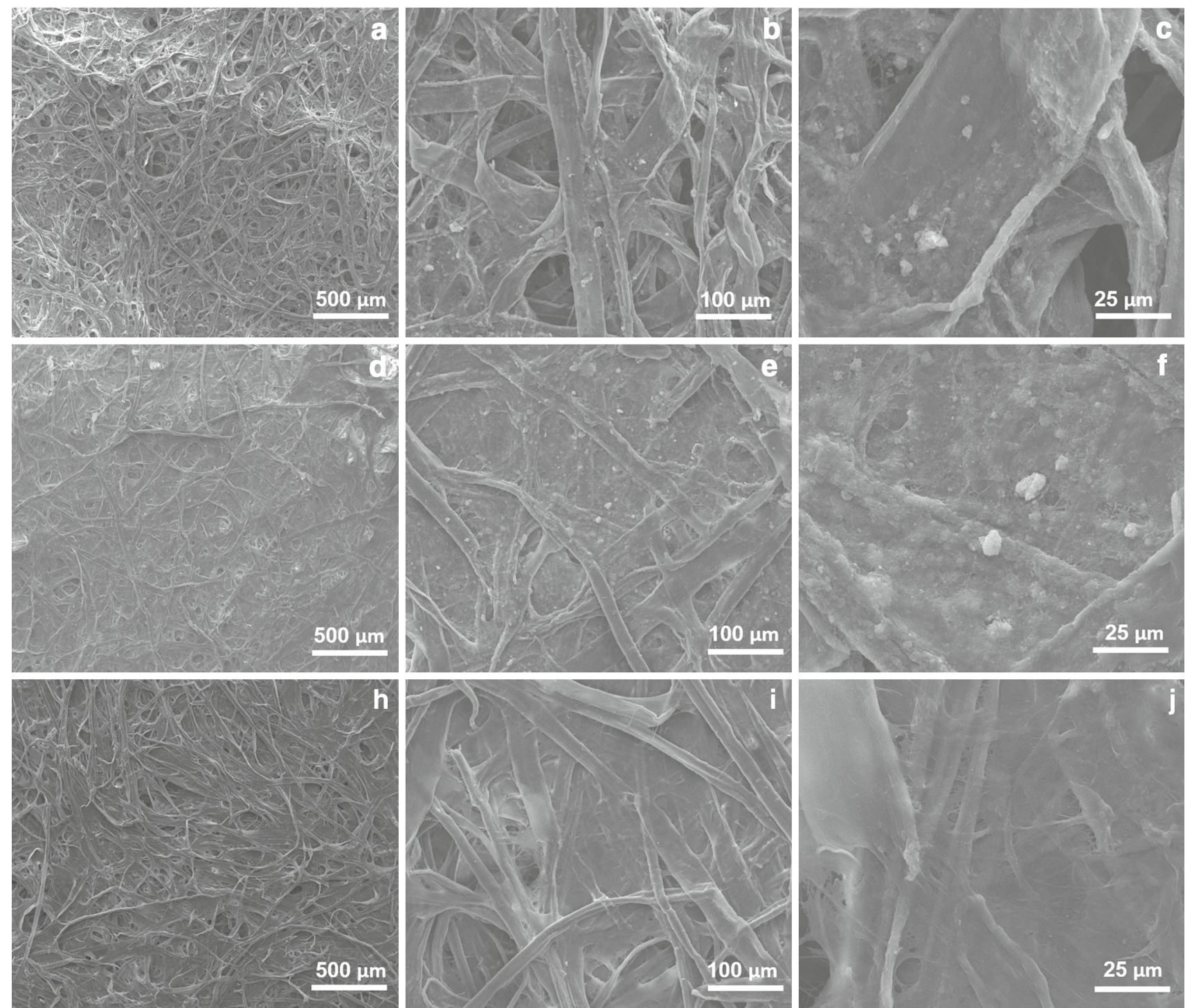

Figure 5. SEM images of $(\mathbf{a}, \mathbf{b}, \mathbf{c}) \mathrm{TiO}_{2}$ photocatalytic paper without loading $\mathrm{PAD},(\mathbf{d}, \mathbf{e}, \mathbf{f}) \mathrm{TiO}_{2}$ photocatalytic paper loaded with $\mathrm{PDA}$, where the mass ratio of PLCF-CF is $1: 1$, and $(\mathbf{h}, \mathbf{i}, \mathbf{j})$ paper prepared by PLCF.

\section{Results and discussion}

\subsection{Characterization of photocatalytic paper}

The loading of PDA on CF by in situ oxidative polymerization of dopamine results in the blackening of the as-prepared photocatalytic paper as shown in figure 2. By comparing the Fourier-transform infrared (FTIR) spectrum of photocatalytic paper loaded with PDA and that without PDA (shown in figure 3), one can find that a number of new absorption peaks assigned to the functional groups, such as catechol and secondary amine of PDA appear in the FTIR spectrum of the paper loaded with PDA. The peak at $1280 \mathrm{~cm}^{-1}$ is attributed to $\mathrm{C}-\mathrm{O}$ stretching vibration of catechol [22]. The peak at $1509 \mathrm{~cm}^{-1}$ corresponds to the $\mathrm{N}-\mathrm{H}$ shearing vibration [23], while the peak at $1554 \mathrm{~cm}^{-1}$ corresponds to $\mathrm{N}-\mathrm{H}$ bending vibration and $\mathrm{C}-\mathrm{N}$ shearing vibration of secondary amine.
The peak at $3212 \mathrm{~cm}^{-1}$ corresponds to $\mathrm{N}-\mathrm{H} / \mathrm{O}-\mathrm{H}$ stretching vibration. Therefore, PDA was successfully attached to $\mathrm{CF}$.

The loading of $\mathrm{TiO}_{2}$ nanoparticles on $\mathrm{CF}$ in preparation of photocatalytic paper was evidenced by XRD technique. The XRD patterns of $\mathrm{TiO}_{2}$ nanoparticles and photocatalytic paper are shown in figure 4 . The diffraction peaks of $\mathrm{TiO}_{2}$ nanoparticles match very well with JCPDS card no. 21-1272. The typical diffraction peaks of anatase $\mathrm{TiO}_{2}$ appear in the curve of $\mathrm{TiO}_{2}$-loaded photocatalytic paper. However, the intensity of diffraction peaks that belong to anatase $\mathrm{TiO}_{2}$ are not as high as pure $\mathrm{TiO}_{2}$ nanoparticles detected in the photocatalytic paper, which may be attributed to the low content of $\mathrm{TiO}_{2}$ nanoparticles based on the $\mathrm{CF}$.

The morphology of as-prepared photocatalytic paper and the distribution of $\mathrm{TiO}_{2}$ nanoparticles in the photocatalytic paper were analysed using scanning electron microscopy (SEM). Figure $5 \mathrm{a}-\mathrm{c}$ and $\mathrm{d}-\mathrm{f}$ shows the $\mathrm{SEM}$ images of $\mathrm{TiO}_{2}$ 

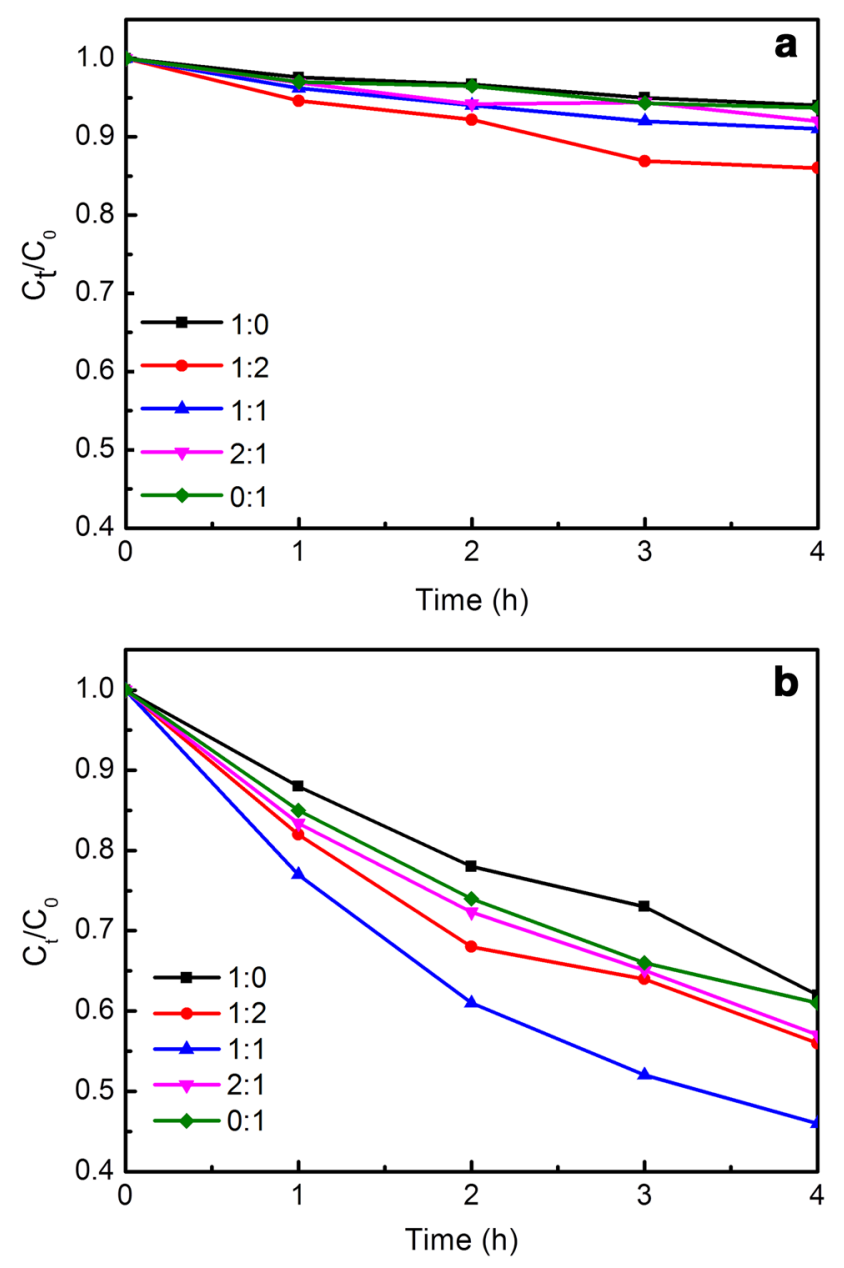

Figure 6. (a) Adsorption and (b) photocatalytic degradation of MO in the presence of photocatalytic paper under the UV light irradiation. The legends are the mass ratio of PLCF-CF in photocatalytic papers.

photocatalytic paper without loading PDA and that loaded with PDA, respectively. The mass ratio of PLCF-CF is $1: 1$ for the PDA loaded paper.

For the photocatalytic paper without loading PDA, there is a clear demarcation between fibres. $\mathrm{TiO}_{2}$ nanoparticles exist as aggregates both in the voids among $\mathrm{CF}$ and on fibre surfaces. The distribution of $\mathrm{TiO}_{2}$ nanoparticles on fibre surfaces is not uniform. Bare $\mathrm{CF}$ without $\mathrm{TiO}_{2}$ nanoparticles can be found in the images. For the photocatalytic paper loaded with PDA, the CF contacts more closely demonstrating that the loading of PDA can significantly increase the bonding among CF. Meanwhile, there are membranes formed on CF surfaces, suggesting the successful coating of in situ formed PDA on $\mathrm{CF}$ surfaces. The boundary between fibres is therefore not obvious due to the occurrence of membranous substances. Furthermore, the $\mathrm{TiO}_{2}$ nanoparticles is more uniformly distributed in the paper than that of the paper without loading PDA although they still occur as aggregates, indicating the retention effect of loaded PDA on $\mathrm{TiO}_{2}$ nanoparticles. However, the porosity of photocatalytic paper is reduced.

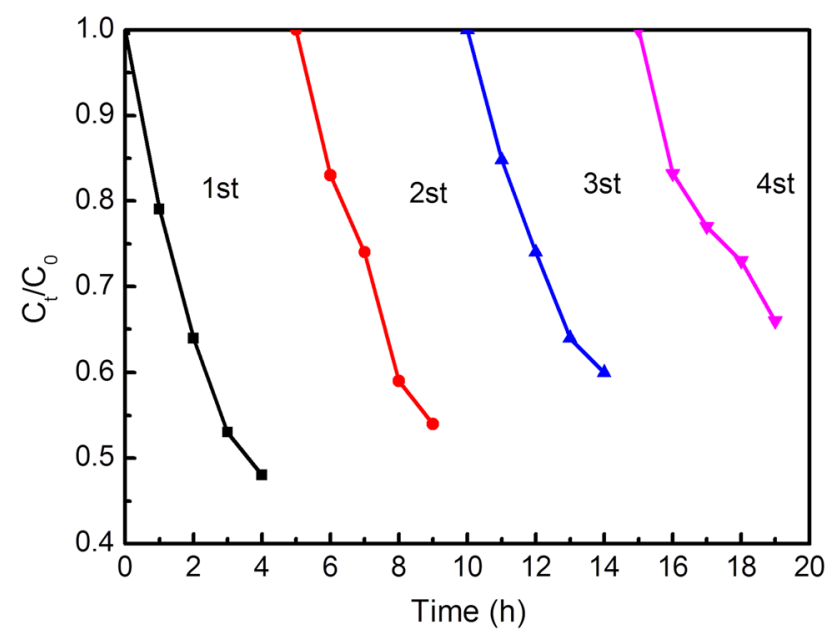

Figure 7. Photocatalytic degradation of MO in the presence of cycled photocatalytic papers with a PLCF-CF mass ratio of 1:1.

To observe more clearly the membrane formed by PDA, paper solely composed of PLCF was manufactured. Figure $5 \mathrm{~h}-\mathrm{j}$ shows the SEM images of paper prepared by PLCF. Apparently, polymeric membranes cover all the $\mathrm{CF}$, making fibre be buried in a layer of polymer. This further confirms that PDA was successfully formed and attached to the CF.

\subsection{Photocatalytic performance of photocatalytic paper}

PDA is an organic conductive polymer [24]. It is assured that the loaded $\mathrm{TiO}_{2}$ nanoparticles can act as active sites to capture light and produce photogenerated electrons and holes under UV light illumination, while the PDA loaded on fibres provides trapping sites to capture the photogenerated electrons released from the $\mathrm{TiO}_{2}$ conduction band, separating the photogenerated electron-hole pairs. This would improve the photocatalytic activity of the $\mathrm{TiO}_{2}$ nanoparticles, accelerating the degradation of MO. The photocatalytic activity and adsorption behaviour of the as-prepared photocatalytic papers with different PLCF-CF mass ratios, were therefore examined. The results are shown in figure 6.

Apparently, all the photocatalytic papers show adsorption for MO in the dark due to their network structures as shown in figure 5. However, the removal rate of MO in the dark is much lower than that under UV light irradiation at the same mass ratio of PLCF-CF, corroborating the photocatalytic activity of the as-prepared photocatalytic paper. The introduction of PLCF may significantly improve the adsorption of MO, such as in the case of introducing one part of PLCF to two parts of $\mathrm{CF}$. The improvement in adsorption of $\mathrm{MO}$ originates from the introduction of amino groups, which increases the attraction with MO on the paper surface. However, introduction of PLCF also reduces the porosity of photocatalytic paper. When the mass ratio of PLCF-CF exceeds 1:2, the adsorption rate of $\mathrm{MO}$ is reduced with the introduction of more PLCF. As a result, the largest adsorption, which can remove $14 \%$ of MO, occurs at the PLCF-CF mass ratio of 1:2. 
As expected, the introduction of PLCF can also significantly improve the photocatalytic activity of photocatalytic paper due to the improvement of $\mathrm{TiO}_{2}$ nanoparticle distribution. The promotion of PDA on the separation of photogenerated electron-hole pairs by capturing electrons released from the $\mathrm{TiO}_{2}$ nanoparticles as previously stated. Therefore, increasing the mass ratio of PLCF-CF can significantly improve the photocatalytic activity of the paper. The removal rate of $\mathrm{MO}$ is increased from 37.8 to $53.9 \%$ after $4 \mathrm{~h}$ of UV irradiation when the mass ratio of PLCF-CF is increased from $0: 1$ to $1: 1$.

However, the introduction of PDA also reduces the porosity of paper by increasing the bonding between fibres, leading to the reduction in adsorption of MO and exposure of $\mathrm{TiO}_{2}$ nanoparticles. More importantly, the loaded PDA shows strong optical absorption as a black conductive polymer, interfering with the optical absorption of $\mathrm{TiO}_{2}$ nanoparticles. Therefore, after the mass ratio of PLCF-CF exceeds $1: 1$, a further increase in the mass ratio results in the decrease of the photocatalytic activity. The photocatalytic paper exhibits its highest photocatalytic performance at the PLCF-CF mass ratio of $1: 1$.

To evaluate the stability of the as-prepared photocatalytic paper in photodegradation of $\mathrm{MO}$, the as-prepared photocatalytic paper with a PLCF-CF mass ratio of 1:1 was used repeatedly. Each cycle lasted for $4 \mathrm{~h}$, and $4 \mathrm{ml}$ of $\mathrm{MO}$ aqueous solution was withdrawn at intervals to determine the extent of MO degradation. The results are shown in figure 7. Obviously, the photocatalytic paper exhibits quite stable photocatalytic activity under UV light irradiation. The photocatalytic activity of the paper is only slightly lowered after it has been reused three times. The removal rate of MO is reduced from over $53 \%$ to about $40 \%$ at the third run of repeated use. One possible reason for the reduction of photocatalytic activity is that when the photocatalytic paper is immersed in water continuously, a small quantity of $\mathrm{TiO}_{2}$ nanoparticles loaded on CF may be washed away, resulting in the loss of photocatalytic activity of the paper. Another possibility is that when used repeatedly, small amount of organic substances may accumulate in the pores of the paper, reducing the exposure of $\mathrm{TiO}_{2}$ nanoparticles.

\section{Conclusion}

Photocatalytic paper with improved photocatalytic activity has been made using $\mathrm{CF}, \mathrm{TiO}_{2}$ nanoparticles and PLCF, which are prepared by in situ oxidative polymerization of dopamine in the presence of $\mathrm{CF}$. $\mathrm{TiO}_{2}$ nanoparticles occur as aggregates and are uniformly distributed on CF surfaces due to the loading of PDA. The photocatalytic activity of the as-made photocatalytic paper is improved with increasing the mass fraction of PLCF when the mass ratio of PLCF-CF is $<1: 1$. When the PLCF-CF mass ratio exceeds $1: 1$, increasing the fraction of PLCF results in the deterioration of photocatalytic activity. The photocatalytic paper thus exhibits the highest photocatalytic performance at the PLCF-CF mass ratio of 1:1. Meanwhile, the photocatalytic paper is rather stable under UV light irradiation. The coating of CF with in situ synthesized PDA and subsequent introduction into paper provide a new approach to prepare photocatalytic paper with improved photocatalytic activity.

\section{Acknowledgements}

The project was funded by the National Natural Science Foundation of China (nos. 31270625 and 21506105).

\section{References}

[1] Zhao Z, Tian J, Sang Y, Cabot A and Liu H 2014 Adv. Mater. 272557

[2] Nam C T, Yang W-D and Duc L M 2013 Bull. Mater. Sci. 36 779

[3] Chen J, Liu W, Li Z and Liu H 2018 Sci. China Mater. 61382

[4] Fujiwara K, Kuwahara Y, Sumida Y and Yamashita H 2017 Langmuir $\mathbf{3 3} 288$

[5] Jiao Y, Wan C and Li J 2017 Funct. Mater. Lett. 101750018

[6] Fukahori S, Iguchi Y, Ichiura H, Kitaoka T, Tanaka H and Wariishi H 2007 Chemosphere 662136

[7] You Y F, Xu C H, Xu S S, Cao S, Wang J P, Huang Y B et al 2014 Ceram. Int. 408659

[8] Malato S, Fernández-Ibáñez P, Maldonado M I, Blanco J and Gernjak W 2009 Catal. Today 1471

[9] Ehsan M A, Khaledi H, Pandikumar A, Huang N M, Arifin Z and Mazhar M 2015 J. Solid State Chem. 23055

[10] Jiang J Y, Xu J L, Liu Z H, Deng L, Sun B and Liu S D 2015 Appl. Surf. Sci. 347591

[11] Li X, Li X, Wang J and Lin S 2015 Sens. Actuators B 21958

[12] Romanos G E, Athanasekou C P, Katsaros F K, Kanellopoulos N K, Dionysiou D D, Likodimos V et al 2012 J. Hazard. Mater. 211-212 304

[13] Hong Y, Yu M, Lin J, Cheng K, Weng W and Wang H 2014 Colloids Surf. B 12368

[14] Li Y, Cai X, Guo J and Na P 2014 Colloids Surf. A 462202

[15] Izadyar S and Fatemi S 2013 Ind. Eng. Chem. Res. 5210961

[16] Zhang J, Liu W, Wang P and Qian K 2013 J. Environ. Chem. Eng. 1175

[17] Lee H, Dellatore S M, Miller W M and Messersmith P B 2007 Science 318426

[18] Liu Y, Ai K and Lu L 2014 Chem. Rev. 1145057

[19] Chen H, Liu W, Hu B, Qin Z and Liu H 2017 Nanoscale 9 18940

[20] Chen H, Liu W and Qin Z 2017 Catal. Sci. Technol. 72236

[21] Gao W, Liu W, Leng Y, Wang X, Wang X, Hu B et al 2015 Appl. Catal. B $\mathbf{1 7 6} 83$

[22] Sureshkumar M and Lee C K 2011 Carbohydr. Polym. 84775

[23] Jiang J H, Zhu L P, Li X L, Xu Y Y and Zhu B K 2010 J. Membr. Sci. 364194

[24] Coskun H, Aljabour A, Uiberlacker L, Strobel M, Hild S, Cobet C et al 2018 Thin Solid Films 645320 